\title{
Interaction Modelling of PCC Flocculation Induced by Cationic Polymer for Water Clarification
}

\author{
Y. S. Peng ${ }^{1}$, A. James ${ }^{1}$ and H. N. Xiao ${ }^{2 *}$ \\ ${ }^{1}$ Department of Chemical Engineering, University of Manchester Institute of Science and Technology, Manchester M60 1QD, UK \\ ${ }^{2}$ Department of Chemical Engineering, University of New Brunswick, Fredericton E3B 5A3, Canada
}

\begin{abstract}
The precipitated calcium carbonate (PCC) has been extensively used as additives in various industrial applications, particularly as fillers for papermaking. Due to the limited retention being achieved in application, it is necessary to clarify the PCC suspension after the application. Cationic polymers are often used as flocculants to induce the aggregation of PCC particles and facilitate the removal of PCC in water clarification. In this work, we focused on identifying the optimum dosage of the polymer and understanding the flocculation mechanism via the characterization of the zeta potential of the PCC suspension. Moreover, the free energy of interaction between PCC particles covered by polymer was investigated using a surface energy modelling that simulated the interaction under various polymer coverages. The modelling results indicated that the electrostatic free energy increased as the surface coverage increased from 33.7 to $81.8 \%$. The model also enables to predict the total free energy and the electrostatic energy as a function of the separation distance in spite of the lack of complete agreement with the experimental observation.
\end{abstract}

Keywords: Cationic polymer, flocculation, free energy, modelling, PCC, Zeta-potential

\section{Introduction}

The precipitated calcium carbonate (PCC) has been extensively utilized in various industrial processes. A typical example is PCC as fillers for papermaking processes, which not only reduces the cost of paper but also improves the quality of the paper. Water clarification for PCC suspension has also become an important task since only limited PCC retention is achieved in application. The major problem encountered in the separation of fine particles (i.e., PCC) from an aqueous medium is the low rates of sedimentation. The poor operating procedures in industry lead to over-design, excessive cost and inefficiency in the effluent plant design. There is therefore tremendous scope for optimization of the parameters inherent to coagulation and flocculation.

Understanding colloidal aggregate characteristics is one of the central issues in many industrial applications. The behaviour of an aggregate of colloidal particles, or floc, in a suspension system is dependent upon its charge or physical characteristics, including surface charge density, zeta potential, size, morphology, and permeability.

The relationship between the measured zeta potential and the double layer structure is still a subject of debate and the shear plane cannot be located with any certainty. Electrokinetics is of immense importance in colloid science, since they give information, however imperfect, which is not obtainable in any other way. There is a clear correlation, for instance,

\footnotetext{
* Corresponding author: hxiao@unb.ca
}

between zeta potential and colloid stability in many cases. Colloidal particles are generally found to be stable if the zeta potential exceeds a certain value and coagulate at lower values. Similarly, deposition of particles on surfaces is very often controlled by the zeta potential of particles or substrates (Eilbeck \& Mattock, 1987). A general mechanism of colloidal flocculation by polymers with high molecular weight has been well documented (Hunter, 1993, Healy \& La Mer, 1964). The process involves the bridging of colloid particles by the polymer chain; hence forming larger structural units that are readily separated from the aqueous dispersing medium. This mechanism is the most probable cause of flocculation with polyelectrolytes (Berlin et al., 1997).

The essential requirements for polymer bridging are that there should be sufficient unoccupied particle surface, allowing the polymer chains to be attached to other particles. To be effective as flocculant, the polymer chains must form extended loops and tails on the surface of substrates, spanning the distance (i.e., double layer thickness) over which interparticle repulsion operates. Also, it is found that there is an optimum dosage range for effective flocculation. At lower dosages, there is insufficient polymer to form adequate bridging links between particles. With excess polymer, there is no longer enough bare particle surface for attachment of segments and the particles become restabilized due to steric repulsion.

The objective of the present work was to investigate the patch mechanism using cationic polyelectrolyte in a PCC system. This is carried out by measuring the surface charge 
density of particles and the free energy of interaction. Both surface charge density and free energy of interaction are determined as a function of polymer dosage in order to assess the effect of polymer addition to the system. The surface charge density is determined using a particle charge detector and particle size measurement. The free energy of interaction between PCC particles is estimated by calculating the electrostatic and van der Waals interactions between particles. The electrostatic energy of interaction is calculated by adapting computer programmes that use finite element analysis to solve the non-linear Poisson-Boltzmann equation. In addition, the relationship between zeta potential and surface potential is also investigated in this PCC system.

\section{Experimental and Modelling Approach}

\subsection{Materials}

Precipitated calcium carbonate (PCC) was a scalenohedral calcite filler product (Specialty Minerals Inc) with an average particle size of $0.8 \mu \mathrm{m}$ and specific surface area of 12 $\mathrm{m}^{2} / \mathrm{g}$. Dispersions of PCC were prepared at concentrations of $5 \% \mathrm{w} / \mathrm{v}$ by mixing PCC powder with $5 \times 10^{-3} \mathrm{M}$ sodium chloride in an agitation vessel for 2 hours. The cationic polymers, Hydrocol 882 (low charge density) and Hydrocpl 864 (medium charge density) were provided by Allied Colloids, UK. Both polymers are acrylamide-based copolymers with high molecular weight. In addition, sodium chloride at a concentration of $5 \times 10^{-3} \mathrm{M}$ is used for stabilizing the ionic strength of the solution.

\subsection{Flocculation and Characterization}

Flocculation of PCC particles was performed through a jar test that has been well received in identifying the optimum dosage of coagulant or flocculant (Hammer, 1998). The turbidity of the PCC was monitored using a turbidity meter (WPA TU1100, U.K.) and presented in the units of NTU (Nephelometric Turbidity Units). The zeta potential of PCC particles was measured using a Zetasizer HS 3000 (Malvern Instruments Ltd., UK). The surface charge of PCC particles or flocs were determined using a particle charge detector Mutek PCD 03 and a PCD titrator (Mutek, Germany).

\subsection{Modelling of the Free Energy of Interaction for a PCC system}

To investigate the effect on the interaction forces and hence the free energy of colloidal particles as a function of polymer dosage, the modelling programme developed in our previous work (James \& Williams, 1985) was adopted and further modified to fit the current systems. The modelling used the finite element method to solve the Poisson- Boltzmann equation regarding the stability of PCC/polymer particles suspended in aqueous solution. The programme consists of two parts, one dealing with the generation of mesh (PENGGEN); and another performing the calculation of the free energy of the system (YSPB2).

\section{Results and Discussion}

\subsection{Optimum Flocculant Dosage}

The work started with experimentally determining the flocculation of PCC induced by cationic polymer or optimising the polymer dosage. One of such results, the optimum dosage of polymer in PCC flocculation, is presented in Figure 1. The polymer used was the medium-charged cationic polymer (Hydrocol 864). The flocculation was performed without adding any acidic or alkaline chemicals.

As can be seen from Figure 1, the residual turbidity decreases as the flocculant dosage increases. However, after reaching the minimum of residual turbidity at $5 \mathrm{mg} / \mathrm{L}$, the further increase in polymer dosage results in a slightly increased residual turbidity, suggesting that the restabilization occurred at this stage.

\section{Total surface charge of PCC}

Further to the effect of polymer dosage on the turbidity of PCC suspension, the total surface charge of PCC as a function of cationic polymer dosage was also determined using polyelectrolyte titration in which sodium salt of polyethylenesulphate (PES-Na) was used as a titrant. The results of the surface charge of PCC particles or flocs in aqueous suspension are shown in Figure 2.

Clearly, the increase in cationic polymer dosage lowers the total surface charge of PCC, which is attributed to the charge neutralization occurred on the PCC surfaces. This result also implies the PCC surface covered by cationic polymer partially.

\section{Zeta-Potential and Surface Coverage}

To further characterize the electrokinetic properties of PCC particles or flocs formed by the cationic polymer at various dosages, the changes in zeta potential with variation of polymer dosage were measured. Consequently, the surface coverage in terms of zeta potential measurement was also estimated accordingly. Results are presented in Table 1.

As shown in the Table, the percentage of total surface coverage increases with the increase in cationic polymer dosage. It is probable that the increase in the polymer dosage will increase the adsorbed amount of cationic polymer on PCC particles, leading to the increase in the surface coverage consequently. The surface coverage corresponding to the optimum polymer dosage (i.e., $5 \mathrm{mg} / \mathrm{L}$ ) is $81.8 \%$, which is not in agreement with the conventional bridging theory, i.e., half surface coverage at optimum dosage (Healy \& La Mer, 1964). It should be noted that there is extreme difficulty in determining the surface coverage due to its complex nature. The surface coverage is influenced by a number of factors including the properties of electrical double layer, the interaction between adsorbed cationic polymer and PCC particle, the ionic strength of solution, and the distribution of adsorbed polymer 


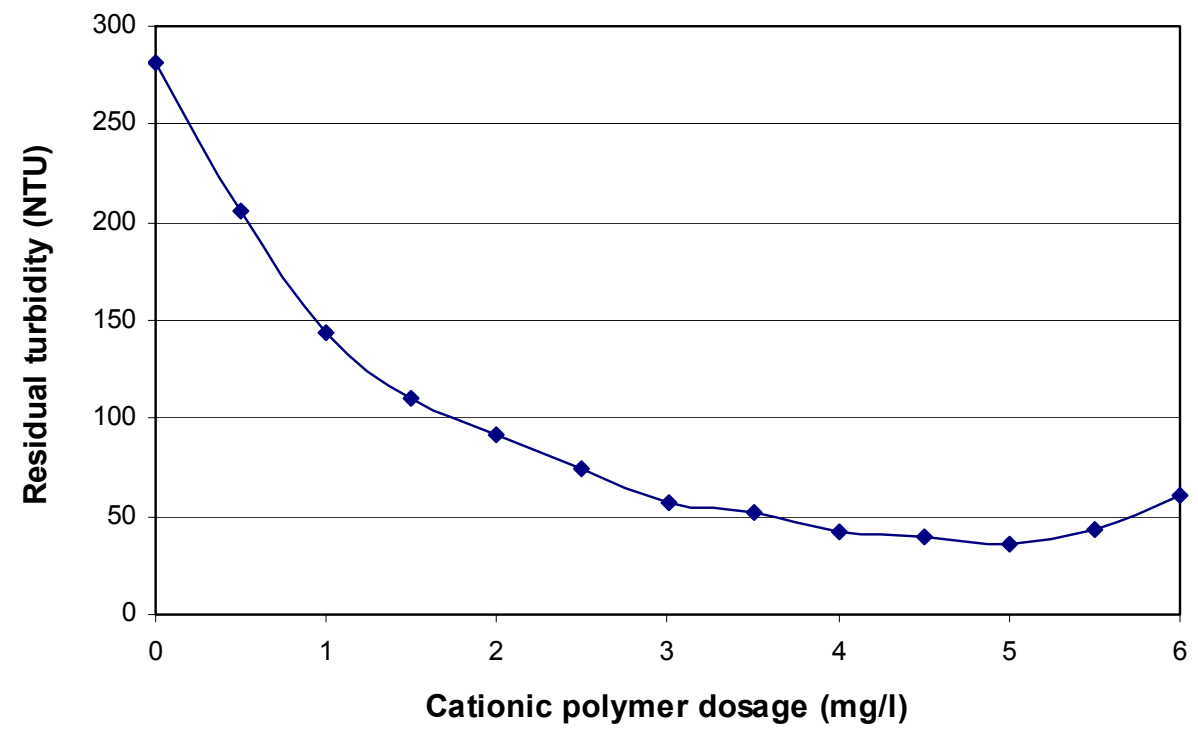

Figure 1. Residual turbidity of PCC suspension as a function of cationic polymer dosage ( $\mathrm{pH}=11.4)$.

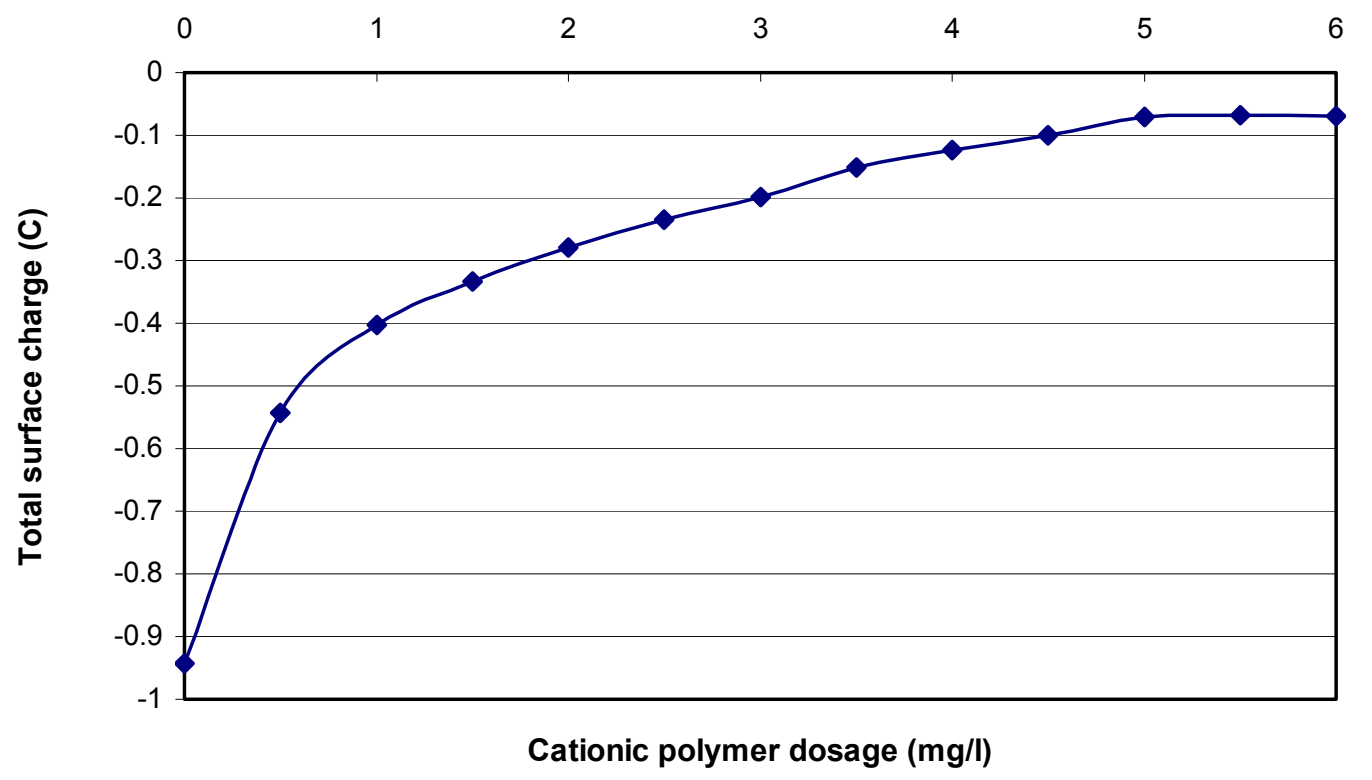

Figure 2. Total surface charge of PCC in suspension as a function of cationic polymer dosage. 


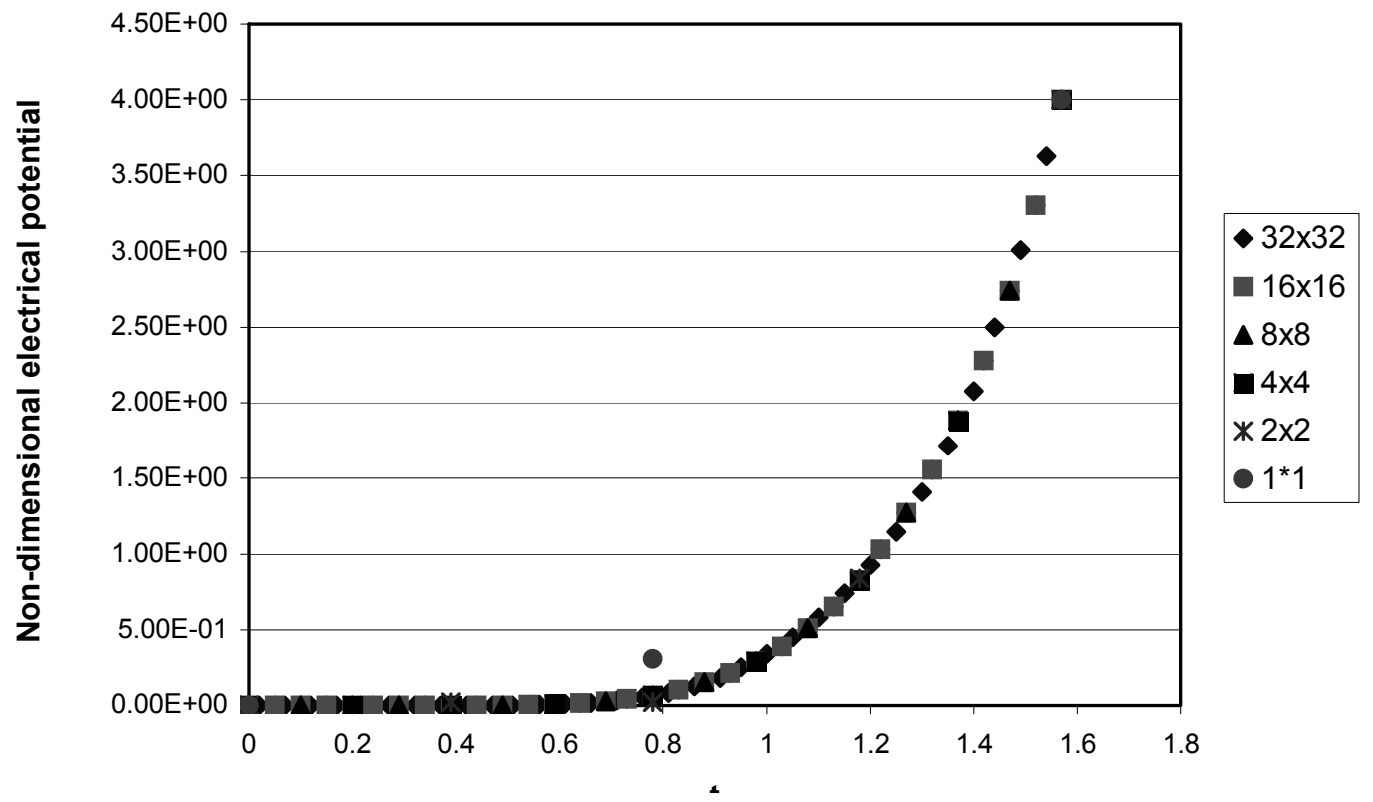

Figure 3. Mesh optimisation for different mesh sizes using the non-dimensional electrical potential at $\mathrm{u}=0$.

on the PCC surface. In some cases, the surface coverage significantly depends on the configuration of the adsorbed polymer chains (Gregory, 1976).

Table 1. Surface Coverage Based on the Calculation of Zeta Potential as a Function of Cationic Polymer Dosage

\begin{tabular}{lll}
\hline Polymer dosage $(\mathrm{mg} / \mathrm{L})$ & $\begin{array}{l}\text { Zeta potential } \\
(\mathrm{mV})\end{array}$ & $\begin{array}{l}\text { \% Surface } \\
\text { coverage }\end{array}$ \\
\hline 0 & -23.8 & 0 \\
0.5 & -18.56 & 22.02 \\
1 & -15.78 & 33.7 \\
1.5 & -13.74 & 42.27 \\
2 & -12.45 & 47.69 \\
2.5 & -10.04 & 57.82 \\
3 & -6.83 & 71.3 \\
3.5 & -6.04 & 74.62 \\
4 & -5.51 & 76.85 \\
4.5 & -4.62 & 80.59 \\
5 & -4.33 & 81.81 \\
5.5 & -4.37 & 91.64 \\
6 & -4.8 & 79.83 \\
\hline
\end{tabular}

\section{Mesh Optimisation for the Computer Model}

In order to explain laboratory results, a successful model should include the important experimental parameters producing the numerical results that are in agreement with the observations. Thus, the model developed in this work intended to reveal the electrokinetic behavior of PCC system under the surface coverage at various polymer dosages. The numerical model was targeted at solving the Poisson-Boltzmann equation for the potential distribution around the PCC particle so that the free energy of the electrical double layer system can be calculated. The mesh generated for the modelling was optimised by evaluating the non-dimensional electrical potential, $\phi$, from the output file for the same conditions but at different mesh sizes. One of the results from mesh optimisation is presented in Figure 3 as an example.

In this Figure, the non-dimensional potential are plotted as a function of variable $t$ for variable $u=0$. It appears that no substantial changes of the output value of $\phi$ for different mesh sizes are found. It is also not practical to increase the mesh size and the experimental duration, as there is no significant change in output. Based on this observation, a compromise decision was taken to consider the mesh size of $20 \times 30$, i.e. 600 elements for all experiments.

\section{Modelling of the Free Energy of Interaction for a PCC System}

There has been significant progress in the description of the interaction between charged colloidal particles in the past decades. In particular, the boundary conditions for solving the Poisson-Boltzmann equation (PBE) governing the potential distribution of a colloidal system have been modified to reflect the physical situation more realistically. In this work, the PCC particles were assumed spherical with approximately the same size. The Poisson-Boltzmann equation was solved in terms of a non-dimensional quantity $\phi$ and a non-dimensional distance $\kappa L_{i}$, where $\kappa$ is the inverse Debye-Length $(\kappa=$ $3.29 \times 10^{9}(I)^{1 / 2}$; $I$ is ionic strength) and $L_{i}$ are any relevant distances in the coordinate system. The non-dimensional quantity $\phi$ is expressed as follows: 
$\phi=e \Psi / k T$

where $e$ is the electrical charge, $\Psi$ is the electrical potential, $k$ is the Boltzmann constant and $T$ is the absolute temperature.

In dealing with the free energy of interacting double layer systems, it is convenient to solve the Poisson-Boltzmann equation in a dipolar coordinate system. The Poisson-Boltzmann equation

$\nabla^{2} \Phi=\frac{1}{2} \sum_{j} c_{j} e^{-z_{i} \Phi}$

is thus transformed to

$\frac{\partial}{\partial t}\left(A(u, t) \frac{\partial \Phi}{\partial t}\right)+\frac{\partial}{\partial u}\left(A(u, t) \frac{\partial \Phi}{\partial t}\right)=\frac{B(u, t)}{2} \Sigma c_{j} e^{-z_{i} \Phi}$

where

$$
\begin{aligned}
& A(u, t)=\frac{\sin u}{\cos t-\cos u} \\
& B(u, t)=\frac{c^{2} \sin u}{(\cosh t-\cos u)^{3}}
\end{aligned}
$$

Equation (1) can be solved numerically using the finite element method (James \& Williams, 1985). In order to simulate partial coverage of the particle surface by polyelectrolyte, it was decided to allocate zones where the zeta potential of the particle has been screened off by the addition of polyelectrolyte, thus variable values of $\phi_{0}$ are specified on $t_{0}$ in the dipolar coordinate system.

The free energy of the double-layer is equal to the amount of work performed in building up the double layer by reversible and isothermal processes. The expression for the free energy around a single colloidal particle is,

$$
G=-\int_{S p} d s \int_{0}^{\Psi_{s}} \sigma_{s}(\Psi) d \Psi a
$$

where $G$ is the total electrostatic free energy around a colloid particle, $\Psi_{\mathrm{s}}$ is the surface potential, $S_{P}$ is the surface area of the particle and $\sigma_{\mathrm{s}}$ is the surface charge density. Equation (2) is inconvenient for numerical computation. Therefore, an equivalent expression for the total energy integral was adopted, which involved only integrals over the volume of the ion atmosphere (Levine 1951):

$G=-\int_{v}(\Delta \Pi+\chi) d v$ where the osmotic pressure difference $\Delta \Pi=k T \sum\left(n_{i}-n_{i}(\infty)\right)$ and the electrostatic stress $\chi=\frac{\varepsilon}{2}|\nabla \Psi|^{2}$; Here $n_{i}$ and $n_{i}(\infty)$ are the mean numbers per unit volume of ions in the double layer and in the bulk fluid respectively. With a dimensionless transformation, the Equation (3) can be further simplified and solved (Sharp \& Honig 1990).

After the optimisation of mesh was performed, the values of the electrostatic free energy of the system as a function of the surface coverage were obtained. Results are shown in Figure 4. As can be seen, the non-dimensional electrostatic free energy for both $5 \kappa a$ and $11 \kappa a$ shows a similar trend. The electrostatic free energy decreases as the surface coverage increases in the range of 0 to $33.7 \%$ coverage; and increases in the range of 33.7 to $81.8 \%$ coverage.

Apparently, the surface coverage by polyelectrolyte affects the repulsive forces between particles. Since the polymer used is a cationic one, the positive charge of the polymer reduces the repulsion between the negatively charged particles of PCC. It seems that the charge neutralisation occur and predominate at the beginning of flocculation. Above the surface coverage at which the minimum electrostatic free energy occurs, the effect of neutralisation diminishes. The increased free energy is likely contributed from the increased electrostatic forces caused by the non-uniform coverage of cationic polymer on the surface of PCC. A similar phenomenon regarding the effect of surface coverage on the nondimensional repulsive free energy was also observed by Al-Ajmi (1998). In addition, negative electrostatic free energies are unexpectedly found in Figure 4 . This might be due to fact that the electrostatic interactions between non-uniformly charged surfaces lead to the net attraction (Sjöström \& Åkesson, 1996; Qian \& Bowen, 1999).

The non-dimensional repulsive free energy shown in Figure 4 can be readily converted to the dimensional repulsive free energy, $V_{R}$, by multiplying $k T N_{A} I / \kappa^{3}$, where $k$ is the Boltzmann constant, $T$ is temperature, $N_{A}$ is Avogadro's number, $\mathrm{I}$ is ionic strength, and $\kappa$ is Debye parameter. As a result, the total free energy (i.e., the sum of the electrostatic (repulsive) free energy of interaction and the attractive free energy of interaction due to van der Waals forces) is obtainable.

The effects of the separation distance and surface coverage on the non-dimensional free energy are shown in Figure 5. From the two bottom curves in the Figure, it is found that the electrostatic energy is significantly lowered at the surface coverage between 33.7 or $47.7 \%$. The electrostatic free energy then increases with increasing surface coverage. Apparently, the results obtained in this case are in agreement with the classic bridging theory proposed by Healy and La Mer (1964) or the best flocculation occurring at around $50 \%$ of surface coverage (Gregory, 1993). However, this does not agree with the current experimental results shown in Figure 1 and Table 1, where the highest surface coverage (81.81\%) corresponds the lowest residual turbidity. 


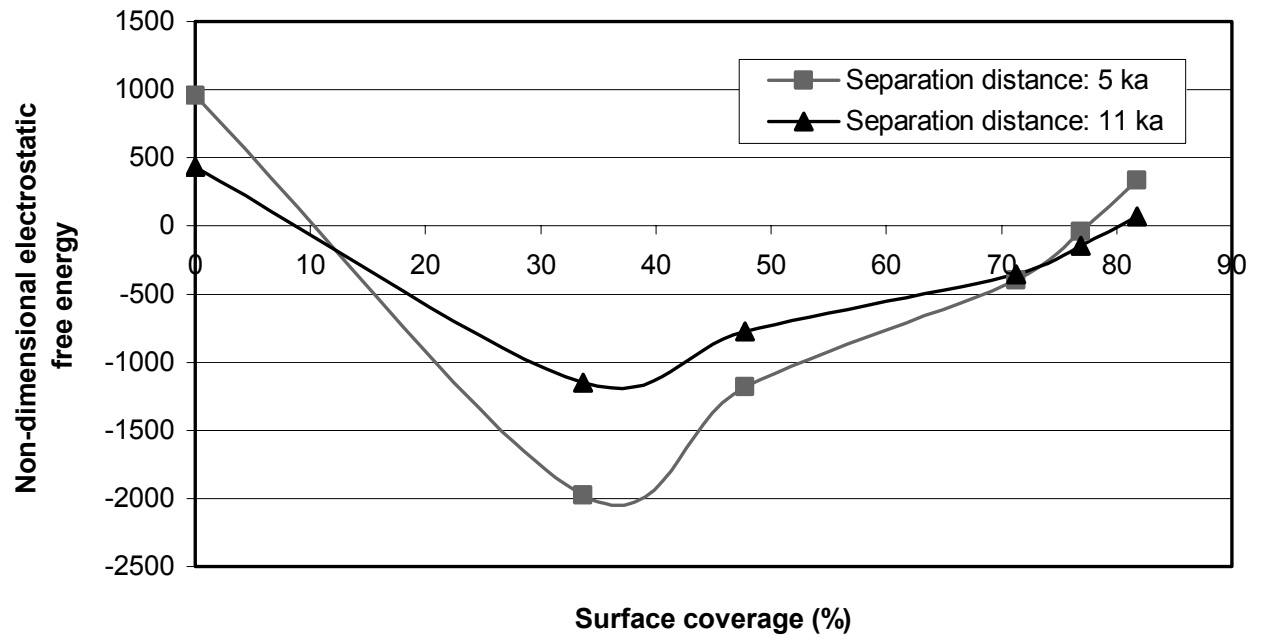

Figure 4. Non-dimensional electrostatic free energy of interaction of the PCC system as a function of surface coverage.

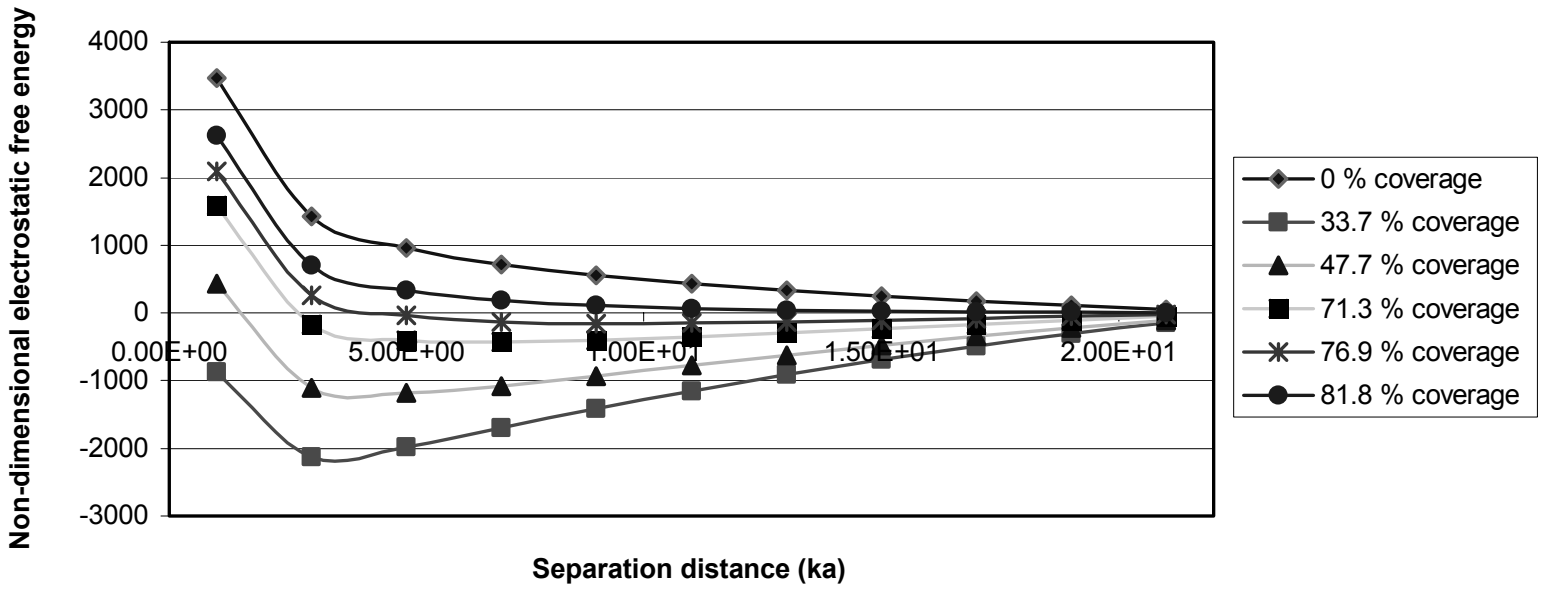

Figure 5. Non-dimensional electrostatic free energy of interaction of the system for PCC particles as a function of surface coverage and particles separation distance. 
The discrepancy in the results could be attributed to a number of influencing factors. In calculating the electrostatic free energy using our numerical model, we assumed that the surface coverage by the polymer was uniformly distributed. However, this is unlikely, and the distribution of adsorbed polymer on the particle surface is likely uneven. Under this circumstance, it is extremely difficult to deal with the distribution of covered surface in estimating the electrostatic free energy. Furthermore, the contact orientation between two covered particles also plays an important role in governing the electrostatic free energy. If two particles covered by polymer approach each other and contact via the sides covered by polymer, the electrostatic free energy will be greater than those contact by the uncovered or partly covered particles. On the other hand, the accuracy of the surface coverage depends on the results of zeta-potential measurements. The values of the zeta-potential are reliable when the single particles of PCC are tested. However, the size of the PCC flocs formed by cationic polymer is substantially larger than that of the single particles of PCC, which could introduce significant errors in zeta-potential measurement due to the limitation of the instrument. Further work on improving the zeta-potential measurements for the flocs is required, which would provide the precise information on the surface coverage.

Although the results obtained from this model are not wholly in agreement with the experimental observations, it provides some preliminary insights into the detailed concepts concerning PCC flocculation. In general, the computer modelling can be a quite sophisticated tool in manipulating particle interactions in the existence of very complicated arrangements like in the case of the present work.

\section{Conclusions}

- PCC particles were effective flocculated by a cationic polymer with medium charge density and high molecular weight. The optimum dosage of the polymer was identified. The zeta potentials of the flocs as a function of polymer dosages were also determined, which leads to the estimation of the surface coverage of PCC particle and provide database for the comparison with the modelling.
- The numerical model was developed via solving the Poisson-Boltzmann equation for the interacting double layer systems. The modelling successfully predicted the electrostatic free energy as a function of the surface coverage. The model also enables to achieve the total free energy and the electrostatic energy as a function of the separation distances between two particles.

The simulation results are consistent with classic flocculation mechanism, but deviated from the findings from the current PCC/cationic polymer system. The reliability in getting zeta-potential, in turn the surface coverage, could be one of the main reasons causing the error. The modelling on flocculation processes not only improves our understanding of the bridging mechanism but also provides a guide for our future research.

\section{References}

Berlin, A., Solomentseva, I.M. and Kislenko, V.N. (1997). Suspension flocculation by polyelectrolyte: Experimental verification of a developed mathematical model. J. Colloid Interface Sci., 191, 273-276.

Eilbeck, W.J. and Mattock, G. (1987). Chemical Processes in Wastewater Treatment, Chichester, Ellis Horwood.

Gregory, J. (1976). The effect of cationic polymers on the colloidal stability of latex particles. J. Colloid Interface Sci., 55, 35.

Gregory, J. (1993). Stability and Flocculation of Suspensions, (Edited by Shamlou, P.A.) Oxford, Butterworth-Heinemann.

Hammer, M.J. (1998). Water and Wastewater Technology, Prentice-Hall, NJ, USA.

Healy, T.W. and La Mer, V.K. (1964). Energies of flocculation and redispersion by polymers. J. Colloid Interface Sci., 19, 323-332.

Hunter, R.J. (1993). Foundations of Colloid Science, Oxford University Press, Oxford.

James, A.E. and Williams, D.J.A. (1985). Numerical solution of Poisson-Boltzmann Equation. J. Colloid Interface Sci., 107, 44-59.

Qian, Y. and Bowen, W.R. (1999). Long-range electrostatic interaction between a charge wall and two similarly charged Colloidal spheres at low surface potential. J. Colloid Interface Sci., 213, 316-321.

Sharp, K.A. and Honing, B. (1990). Calculating total electrostatic Energy with the non-Linear Poisson-Boltzmann Equation. $J$. Phys. Chem., 94, 7684-7692.

Sjöström, L. and Åkesson, T. (1996). The stability of charged colloids-attractive double layer forces due to asymmetric charge distribution. J. Colloid Interface Sci., 181, 645-653. 